\title{
An enhanced energy-efficient routing protocol for wireless sensor network
}

\author{
Ikram Daanoune, Abdennaceur Baghdad, Abdelhakim Ballouk \\ Department of Electrical Engineering, Laboratory of Electronics, Energy, Automatics and Data Processing, \\ Faculty of Sciences and Techniques of Mohammedia, Hassan II University of Casablanca, Morocco
}

\begin{tabular}{l} 
Article Info \\
\hline Article history: \\
Received Feb 22, 2020 \\
Revised Apr 25, 2020 \\
Accepted May 7, 2020
\end{tabular}

\section{Keywords:}

Clustering

Current energy

LEACH

Network lifetime

WSN

\begin{abstract}
Recent few years, Wireless Sensor Network (WSN) has been an increasingly important technology that has been applied in almost all domains, even in complex environments where human activity is impossible. In WSN, various factors are impacted energy consumption, such as communication protocols, packet data transmission, and limited battery. So, the lifespan of the WSNs is limited. In this context, energy efficiency is the factor most attracted by many researchers. In this paper, we proposed a new improved LEACH routing protocol. This proposed protocol based on the current energy to select cluster-heads, and it uses a root cluster-head with more current energy and low distance to the sink to gather all data, then sends it to the sink. The simulation results in MATLAB confirmed that the proposed algorithm performed better than the conventional LEACH protocol, and increased the network lifetime in WSN.
\end{abstract}

Copyright (c) 2020 Insitute of Advanced Engineeering and Science. All rights reserved.

\section{Corresponding Author:}

Ikram Daanoune,

Department of Electrical Engineering,

Laboratory of Electronics, Energy, Automatics and Data Processing (EEA\&TI), Hassan II University,

FST Mohammedia-Casablanca (B.P. 146 Mohammedia 20650 Morocco).

Email: daa.ikram@gmail.com

\section{INTRODUCTION}

In the last few years, WSN recognizes an increased interest through its use in different domains such as military, medical, environment, etc. [1,2]. The WSN consists of a large number of low power microsensor nodes deployed in a large space with at least one BS [3]. Every micro-sensor monitors physical or environmental conditions such as pressure, temperature, humidity, etc. [4] then dispatch the collected data back to the BS. In WSN, the positions of the nodes are not predetermined, which allows an autonomous organization of the network [5,6]. As shown in Figure 1 WSN includes sensor nodes distributed randomly, a BS receives all data that was collected from the environment, and the user that gathered data through the internet.

Each sensor node in the network has four units: sensing, treatment, wireless transmission and power unit (generally are batteries) [7] as presented in Figure 2. One of the most important units is the power unit. Sensor nodes in such an environment are energy-constrained since the batteries can not be recharged or replaced [3]. Consequently, designing an energy-aware protocol got an interest to prolong the network lifetime [8]. Thus, energy consumption is the major important factor in most applications where all sensor nodes are constrained with energy which related to the lifetime of the network. The restricted power of nodes needs the design of a protocol of communication that conserves energy [9]. The restricted power of nodes needs the design of a protocol of communication that conserves energy [9]. In this way, many kinds of research are focused on routing protocols, which is one of the most technologies in WSNs. 


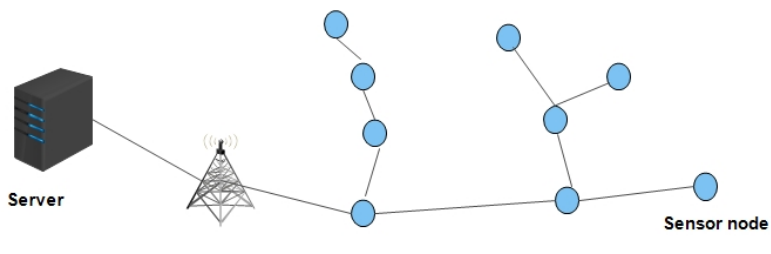

Base Station

Figure 1. WSN architecture

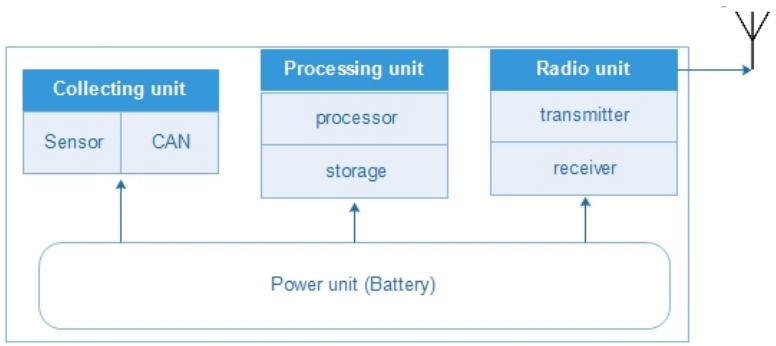

Figure 2. Sensor node units in WSN

In the literature, many routing protocols have designed to arrange the transmission of data from nodes to the sink [10], and most of them are based on the hierarchical clustering mechanism [11]. The Low Energy Adaptive Clustering Hierarchical (LEACH) protocol [12] is the famous and the popular hierarchical clustering routing protocol, which will be described in the next section [13]. In order to prolong the network lifetime and reduce energy consumption in LEACH, various protocol are proposed, such as DMR [14], MH-LEACH [15], MHT-LEACH[16], O-LEACH [17], etc. In this work, we are based on the LEACH protocol to develop a new enhanced LEACH protocol, which improves the energy consumption in WSNs. The present paper aims to propose energy-efficiency, stability, residual energy, distance, and the multi-hop technique based clustering routing protocol. This protocol uses these metrics to minimize the energy consumption in the network and extend the network lifespan in WSNs.

The remaining of the paper is orderly as follows: Section 2 presents the LEACH protocol. The proposed protocol will be described in section 3. Section 4 covers results and analysis. Finally, the last section concludes the paper.

\section{LEACH (LOW ENERGY ADAPTIVE CLUSTERING HIERARCHY)}

LEACH [12] is the most popular and the most famous clustering hierarchical protocol designed for an energy economy of the network [18]. It uses the clustering technique which divides the network into clusters, and through clustering mechanism, the nodes organize themselves into a hierarchical organization. Every round in the LEACH algorithm consists of two main phases: set-up phase and steady phase[19].

In the first phase, $\mathrm{CHs}$ are elected and clusters are formed. At the beginning of each round, every sensor node produces a random value between 0 and 1 [20]. If this value is lower than the probability function $\mathrm{T}(\mathrm{n})$ defined in the equation (1), this node selected as CH. Even if, it becomes a normal node [21].

$$
T(n)=\left\{\begin{array}{l}
\frac{P}{1-P *(r \bmod (1 / P))}, n \in G \\
0 \quad \text { else }
\end{array}\right.
$$

Where $\mathrm{p}$ concerned the percentage of the number of clusters in the network. $\mathrm{r}$ is the current epoch, and $\mathrm{G}$ is a group of nodes that have not been selected as $\mathrm{CHs}$ in epoch $\mathrm{r}$.

Afterward, the $\mathrm{CHs}$ are selected, they diffuse information to the network. Therefore, each normal node chooses its $\mathrm{CH}$ belongs depending on the strength of the received signal. In the second phase, when clusters are configured and each node knows its time slot in TDMA. The normal nodes transmit their collected data to their $\mathrm{CHs}$ by their time slots in the TDMA schedule. The TDMA schedule is used to adjust the channel of internal 
access in every cluster and reduce the interference between clusters [22]. Therefore, each $\mathrm{CH}$ aggregates and compresses the received data from its members with its own. Thus, the cluster's information is routed directly in a single hop to the BS through the CHs.

\subsection{Radio energy consumption model}

In each node, the transmitter consumes $E_{T x}$ defined in the equation (2) to transfer $n$ bits remote receiver di meters. There are two different radio models: (1) the free space (fs) that it is adopted when the distance between the transmitter and the receiver is less than the threshold distance $d i_{0}$; (2) the multi-path (mp) which it is used when the distance is greater than $d i_{0}$. Then, the receiver consumes $E_{R x}$ described in the (4) to receive $\mathrm{n}$ bit of packet.

$$
\begin{aligned}
& E_{T x}(n, d i)=\left\{\begin{array}{l}
n \cdot E_{\text {elec }}+n \cdot \epsilon_{f s} \cdot d i^{2}, \quad d i<d i_{0} \\
n \cdot E_{\text {elec }}+n . \epsilon_{m p} \cdot d i^{4}, \quad d i>=d i_{0}
\end{array}\right. \\
& d i_{0}=\sqrt{\frac{\epsilon_{f s}}{\epsilon_{m p}}} \\
& E_{R x}(n)=n * E_{\text {elec }}
\end{aligned}
$$

Where the energy consumption of the transmitter or the receiver is introduced by $E_{\text {elec }}, \epsilon_{f s}$ and $\epsilon_{m p}$ present the energy consumption factor of amplification in the free space and the multi-path respectively.

LEACH is designed to decrease energy consumption. It has some advantages, such as every node can equally divide the charge imposed at $\mathrm{CHs}$ to a certain extent because any sensor node that was selected as a $\mathrm{CH}$ in some epoch can not be reselected as a $\mathrm{CH}$ yet [23]. Besides, the exploitation of the TDMA avoids the collision between $\mathrm{CHs}$. Nonetheless, it has some disadvantages as it affects the single-hop while $\mathrm{CHs}$ communicate directly to the sink. Furthermore, for long-distance betwixt $\mathrm{CH}$ and the BS can produce more energy consumption that makes LEACH not apt for large networks. Moreover, the random selection of $\mathrm{CHs}$ leads to not giving all nodes the possibility to become CHs. Thereby, if a node with less current energy is elected as $\mathrm{CH}$ would die rapidly, which affects the network life cycle.

\section{THE PROPOSED PROTOCOL}

In this document, our contribution is an enhanced LEACH algorithm. The overall purpose of the proposed protocol is selecting $\mathrm{CH}$ according to the residual energy of nodes to avoid the participation of nodes with less energy as $\mathrm{CH}$. To prevent that all $\mathrm{CHs}$ communicate with the $\mathrm{BS}$, it picks out a $\mathrm{CH}$ with the greatest residual energy and the smallest distance to the sink as a parent $\mathrm{CH}$. Then, it uses the multi-hop between $\mathrm{CHs}$ to reach the parent $\mathrm{CH}$ as we can see in Figure 3. The multi-hop technique is used to improve the transmission distance betwixt $\mathrm{CHs}$ and the sink. It includes two principal phases as mentioned below. Set-up phase where we form clusters and we select $\mathrm{CH}$ of each cluster. The steady phase that contains three sub-phases.

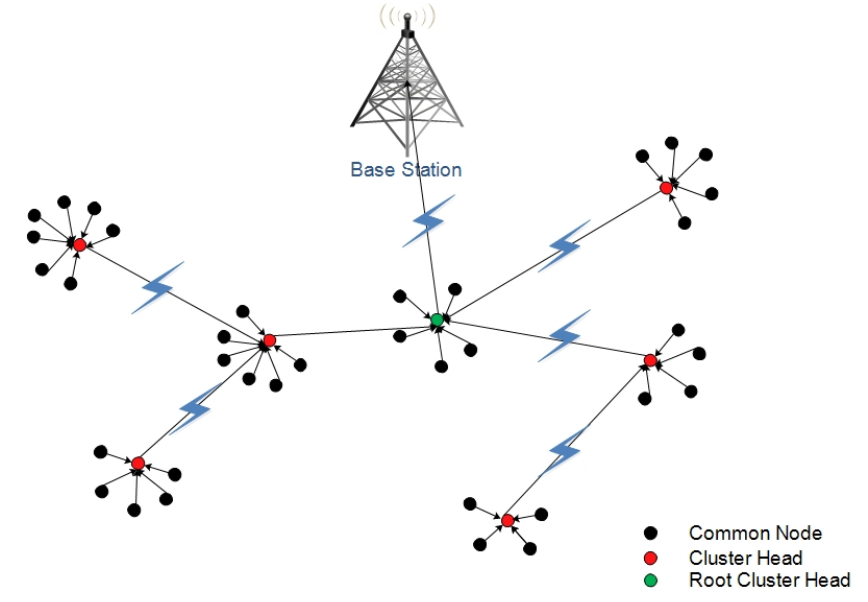

Figure 3. The proposed protocol architecture 


\subsection{Set-up phase}

The random selection of CHs in LEACH leads to not giving all nodes the opportunity to become $\mathrm{CHs}$, also it results in the energy imbalance of sensor nodes. To guarantee the energy balance in the network, the proposed algorithm selects $\mathrm{CH}$ according to the current energy. In every epoch, each node computes its current energy applying the (5). This node can participate in the $\mathrm{CH}$ role if its current energy is superior to the average current energy.

$$
E_{\text {cur }}=E_{\text {init }}-E_{\text {con }}
$$

Where

$$
E_{c o n}=E_{T x}+E_{R x}
$$

$E_{T x}$ and $E_{R x}$ are defined respectively in equation (2) and (4) in section 2.1., and $E_{\text {init }}$ is the initial energy provides to the nodes at the beginning.

Thus, based on $[12,24,25]$ we defined a new threshold function as shown in (6).

$$
T h(n)=\left\{\begin{array}{ll}
\frac{P E}{1-P_{r} *(t \%(1 / P r))}, n \in C & \text {, Where }
\end{array} \quad \text { PE }=\operatorname{Pr} * \frac{E_{\text {cur }}}{E_{\text {init }}}\right.
$$

Where the percentage of the number of CHs in the network is presented by $P_{r}, \mathrm{t}$ introduces the number of the current turn, $\mathrm{C}$ presents the collections of the nodes that have not yet been $\mathrm{CH}$ in the last $1 / P_{r}$ turns. $E_{\text {init }}$ represents the initial energy provides the nodes at the beginning whereas $E_{c u r}$ shows the current energy of nodes at the $\mathrm{t}$ turn.

After the first condition, each node takes a random value between 0 and 1 . This node will be elected as a $\mathrm{CH}$ at the current turn if this value is inferior to the threshold function Th. On the other hand, it becomes a normal node. Afterward, $\mathrm{CHs}$ broadcast information to the network. Each normal node determines its $\mathrm{CH}$ belongs according to the strength of the received signal from $\mathrm{CH}$.

\subsection{Steady phase}

\subsubsection{TDMA (time divisionmultiple access) schedules}

TDMA schedule is created by every $\mathrm{CH}$ after cluster configuration according to the distance and the total number of nodes in each cluster [25, 26]. Each normal node has a TDMA time slot for sending its data to its $\mathrm{CH}$.

\subsubsection{Root cluster head selection}

In this phase, the proposed algorithm selects the root $\mathrm{CH}$ that has residual energy more than the average residual energy of $\mathrm{CHs}$ and its distance to the sink is less than the average distance between CHs and the sink, where are depicted in (7) and (8) respectively.

$$
\begin{gathered}
E_{\text {average }}=\frac{\sum_{i=1}^{C H} E_{c u r}(i)}{C H} \\
d_{\text {average }}=\frac{\left.\sum_{j=1}^{C H} d_{(} j\right)}{C H}
\end{gathered}
$$

$E_{\text {cur }}$ offers the residual energy of $\mathrm{CH}$ at the current turn when $d$ indicates the distance between every $\mathrm{CH}$ and the sink.

\subsubsection{Data transmission}

When all components in the network know their roles in the previous phases, the normal nodes starting to gather data from the environment then transfer it to their $\mathrm{CHs}$ corresponding. Afterward, each $\mathrm{CH}$ communique with the closest $\mathrm{CH}$ to reach the root $\mathrm{CH}$ applying the multi-hop technique. After receiving information of all $\mathrm{CHs}$, the root $\mathrm{CH}$ aggregates them with its own then sends them directly in a single hop to the sink as shown in Figure 3. 


\section{RESULT AND ANALYSIS}

In this section, we use simulation to analyze and emulate the performance of the proposed approach. Simulations are performed in the Matlab 2016b platform. The simulation parameters of our proposed model are mentioned in Table 1. We deployed randomly 200 nodes in area $200 * 200 \mathrm{~m}^{2}$, the sink was placed at position $(100,250)$.

Table 1. Parameters value

\begin{tabular}{lrr}
\hline Parameter & Symbol & Value \\
\hline Total number of nodes & $\mathrm{N}$ & 200 \\
Total number of sinks & $\mathrm{S}$ & 1 \\
Area & $\mathrm{M} * \mathrm{~N}$ & $200 * 200 \mathrm{~m}^{2}$ \\
Initial energy & $E_{\text {init }}$ & $1 \mathrm{Joule}$ \\
The sink location & $(\mathrm{x}, \mathrm{y})$ & $(100,250)$ \\
The deployment of nodes & deployment & $\mathrm{random}$ \\
Energy consumed in the Radio module to transmit or receive the signal & $E_{T x}=E_{R x}$ & $50 \mathrm{~nJ} / \mathrm{bit}$ \\
Energy consumed by the amplifier to transmit at a short distance & $\epsilon_{f s}$ & $10 \mathrm{pJ} / \mathrm{bit}_{\mathrm{m}} \mathrm{m}^{2}$ \\
Energy consumed by the amplifier to transmit at a longer distance & $\epsilon_{m p}$ & $0.0013 \mathrm{pJ} / \mathrm{bit} / \mathrm{m}^{4}$ \\
Data aggregation energy & $\mathrm{EDA}$ & $5 \mathrm{~nJ} / \mathrm{bit}$ \\
Packet size & $\mathrm{n}$ & $500 \mathrm{bytes}$ \\
Number of rounds & $\mathrm{r}$ & 6000 \\
\hline
\end{tabular}

The main purpose of the proposed algorithm is to extend the network lifetime by selecting CHs according to the current energy and choosing a root $\mathrm{CH}$. In the beginning, all nodes have the same initial energy $E_{\text {init }}=1$ Joule. Each sensor node transmits a 4000 bits data packet per round to its cluster head. The percentage of the total number of $\mathrm{CHs}$ in the network is $5 \%$, while the simulation is repeated with $10 \%$ and $20 \%$ both for the proposed algorithm and LEACH.

The simulation result that shows the relative performance of discussed protocols with parameter values of Table 1, and $P_{r}=20 \%$ are presented in Figure 4 (a). It provides dead nodes depending on rounds. Figure 4 (b) compares the behavior of both LEACH and the proposed protocol for $P_{r}=10 \%$. Figure 5 illustrates the diagram of comparative analysis of both discussed protocols for $P_{r}=5 \%$, while FND presents the First Die Node, and HND indicates the Half Node Die. Figure 6 (a), Figure 6 (b), and Figure 7 illustrate the total current energy of nodes in the network for $20 \%, 10 \%$, and $5 \%$ respectively.

The obtained results from the tree cases exhibit the effectiveness of our method for optimizing the energy consumption in WSNs. Thereby, it indicates that the proposed algorithm has performed better than the original LEACH protocol in all cases by balancing the total energy consumption, increasing the stability period and extending the network lifetime.

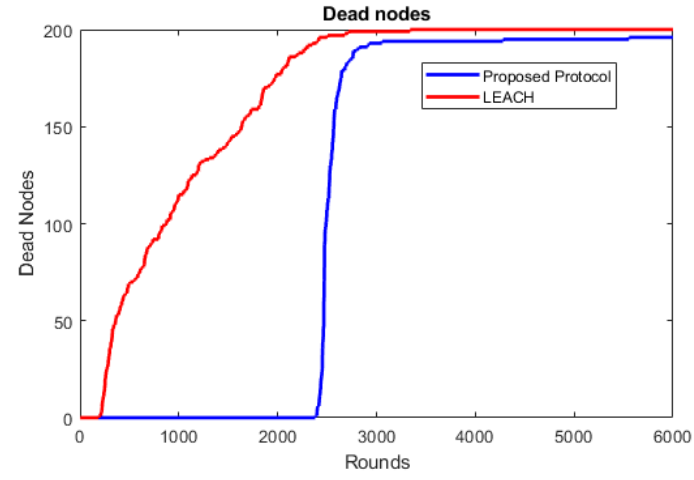

(a)

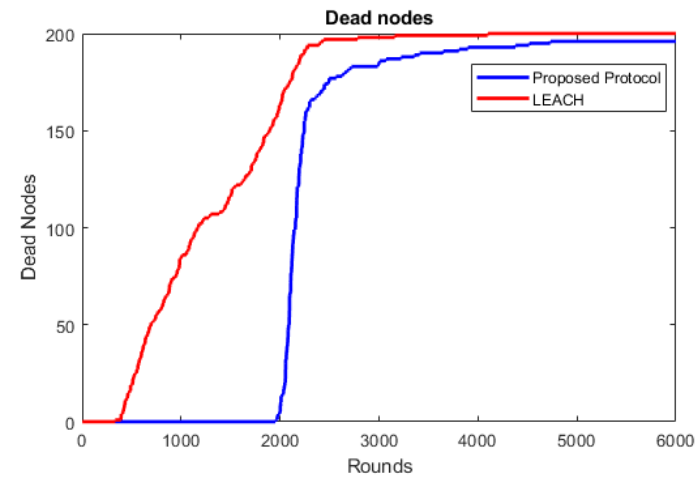

(b)

Figure 4. Dead nodes in LEACH and the proposed protocol for $20 \%$ and $10 \%$, (a) Dead nodes for $20 \%$, and (b) Dead nodes for $10 \%$ 


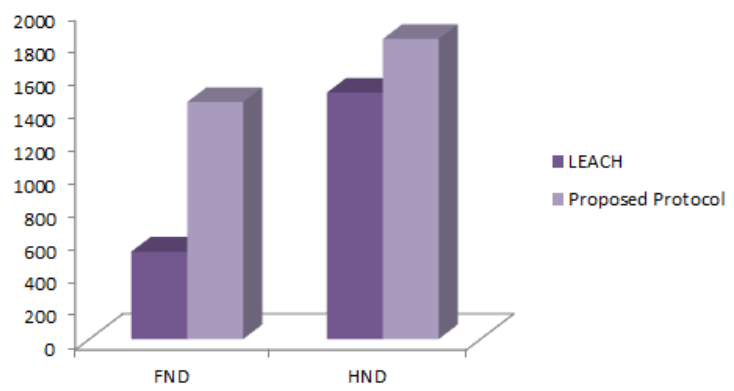

Figure 5. Dead nodes in LEACH and the proposed protocol for 5\%

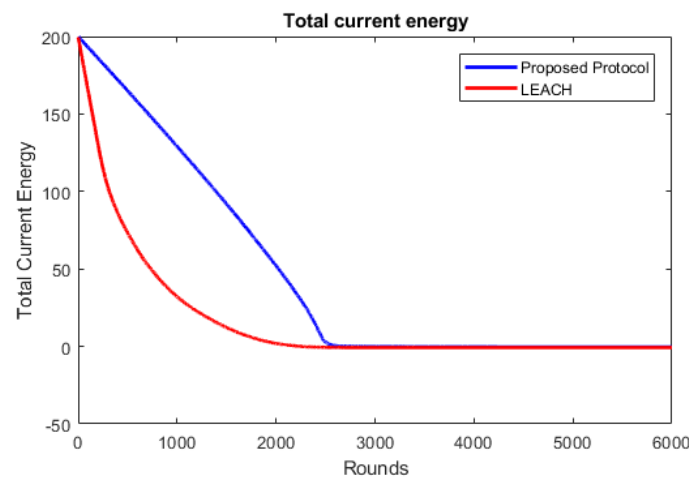

(a)

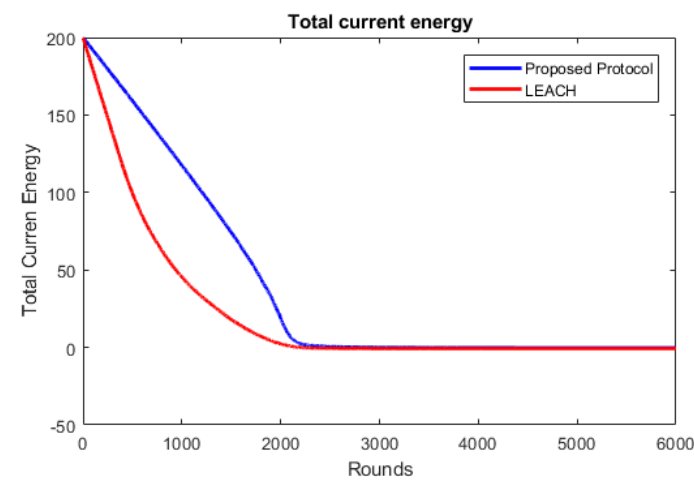

(b)

Figure 6. Total current energy in LEACH and the proposed protocol, (a) Total current energy for 20\%, and (b) Total current energy for $10 \%$

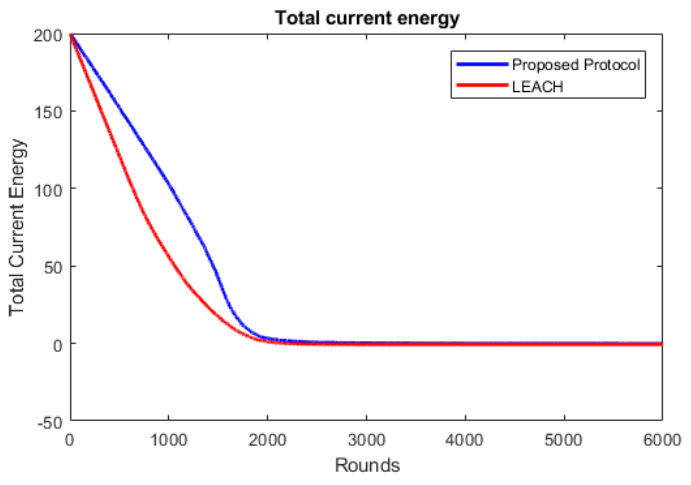

Figure 7. Total current energy in LEACH and the proposed protocol for $5 \%$

\section{CONCLUSION}

Presently, the major challenge faced for routing protocol in WSN is making an energy-efficient design. To solve this problem, it must make the sensor node working for a long period with less consumption of energy. The sending data and reception present generally the major energy consumption in the network. To meet this challenge several clustering routing protocols have been developed. This paper proposed a new approach for hierarchical clustering routing protocol in WSN with energy-saving by the hierarchical and clustering method. The first purpose of the enhanced protocol is balancing the energy consumption of nodes. The second purpose is prolonging the network lifetime and improving the stability period through: (1) taking into account the 
current energy in $\mathrm{CH}$ selection, (2) consideration of current energy and the distance to the sink to pick a leader $\mathrm{CH}$ that send aggregated data to the sink after receiving all collected data. The present study was designed to evaluate the proposed protocol performance against the original LEACH algorithm.

Simulation results in MATLAB confirmed that the proposed algorithm enhances the stability period. Hence, it extends the network lifespan more than the original LEACH protocol owing to the number of dead nodes and the total current energy of the network. Thus, it indicates also that the proposed algorithm achieves better results than the conventional LEACH protocol in all discussed cases.

As future research, it is destined to implement other QoS (Quality of Service).

\section{ACKNOWLEDGEMENTS}

The authors of this work would like to appreciate the excellent suggestions of the reviewers and editors of the IJECE journal to improve the quality of this document.

\section{REFERENCES}

[1] R. Rai and P. Rai. "Survey on Energy-Efficient Routing Protocols in WSN Using Game Theory," Advances in Communication, Cloud, and Big Data, vol. 31, pp.1-9, 2019.

[2] I. Daanoune, A. Baghdad, and A. Balllouk, "A comparative study between ACO-based protocols and PSO-based protocols in WSN," 7th Mediterranean Congress of Telecommunications, pp. 1-4, 2019.

[3] S. Shanthi, P. Nayak, and S. Dandu, "Minimization of Energy Consumption in WSN by Using a Special Mobile Agent," Immunological Tolerance, vol. 1899, pp. 359-368, 2019.

[4] Mohammed Al-Shalabi, Mohammed Anbar, Tat-Chee Wan, and Ahmad Khasawneh, "Variants of the Low-Energy Adaptive Clustering Hierarchy Protocol: Survey, Issues and Challenges," Electronics, vol. 7, no. 8, pp. 136, 2018.

[5] M. Haque, T. Ahmad, and M. Imran, "Review of Hierarchical Routing Protocols for WSN," Intelligent Communication and Computational Technologies, vol. 19, pp. 237-246, 2018.

[6] Heena Dhawan and Sandeep Waraich, "A Comparative Study on LEACH Routing Protocol and Its Variants in Wireless Sensor Networks: A Survey," International Journal of Computer Applications, vol. 95, no. 8, pp. 21-27, 2014.

[7] P. Dubey, S. Veenadhar, and S. Gupta, "Survey on Energy Efficient Clustering and Routing Protocols of Wireless Sensor Network," International Journal of Scientific Research, vol. 5, no. 1, 2019.

[8] Ameer Ahmed Abbasi and Mohamed Younis, "A survey on clustering algorithms for wireless sensor networks," Computer Communications, vol. 30, no. 14-15, pp. 2826-2841, 2007.

[9] Hicham Ouldzira, Hajar Lagraini, Ahmed Mouhsen, Mostafa Chhiba, and Abdelmoumen Tabyaoui, "MG-leach: an enhanced leach protocol for wireless sensor network," International Journal of Electrical and Computer Engineering, vol. 9, no. 4, pp. 3139-3145, 2019.

[10] Emad A. and Ion M. "IMHT: Improved MHT-LEACH protocol for wsn," 8th International Conference on Information and Communication Systems, pp. 246-251, 2017.

[11] P. Tyagi and V. Verma, "Leach Routing Protocol For Wireless Sensor Networks: A Survey," Journal of Advances in Computational Intelligence and Communication Technologies, vol. 2, no. 1, pp. 56-59, 2018.

[12] W. R. Heinzelman, A. Chandrakasan, and H. Balakrishnan, "Energy-efficient communication protocol for wireless microsensor networks," 33rd Annual Hawaii Inter. Conf. on System Sciences, vol. 1, 2000.

[13] Ravi Kishore Kodali and Naveen Kumar Aravapalli, ”Multi-level LEACH protocol model using NS-3," IEEE International Advance Computing Conference, pp. 375-380, 2014.

[14] Emad Alnawafa and Ion Marghescu, "New Energy Efficient Multi-Hop Routing Techniques for Wireless Sensor Networks: Static and Dynamic Techniques," Sensors, vol. 18, no. 6, 2018.

[15] J. H. B. Neto, A. R. Cardoso, and J. Celestino Jr, "MH-LEACH: A Distributed Algorithm for Multi-Hop Communication," Wireless Sensor Networks, vol. 2014, pp. 55-61, 2014.

[16] Emad A. and Ion M, "MHT: Multi-hop technique for the improvement of leach protocol," 15th RoEduNet Conference: Networking in Education and Research, pp. 1-5, 2016.

[17] A. El Aalaoui and A. Hajraoui, "Energy efficiency of organized cluster election method in wireless sensor net- works," Indonesian Journal of Electrical Engineering and Computer Science, vol. 18, no. 1, 2020.

[18] A. S. Kirsan, M. U.H Al Rasyid, and I. Syarif, "Efficient Energy for Cluster Head Selection using New LEACH- based routing protocol in WSN," International Electronics Symposium (IES), pp. 70-75, 2019. 
[19] A. Yektaparast, Fatemeh-Hoda Nabavi, and A. Sarmast, "An Improvement on LEACH Protocol (CellLEACH)," 14th international conference on advanced communication technology, pp. 992-996, 2012.

[20] S. Smys, R. Bestak, J.I.Z. Chen, and I. Kotuliak, "Inter. Conference on Computer Networks and Communication Technologies," Lecture Notes on Data Engineering and Communications Technologies, 2019.

[21] Chunyao Fu, Zhifang Jiang, Wei Wei, and Ang Wei, ”An Energy Balanced Algorithm of LEACH Protocol,' WSN, vol. 10, no. 1, 2013.

[22] Seddiki Noureddine, Benahmed Khelifa, and Belgachi Mohammed, "Approach to minimizing consumption of energy in wireless sensor networks," International Journal of Electrical and Computer Engineering, vol. 10, no. 3, 2020.

[23] Xuxun Liu, "A Survey on Clustering Routing Protocols in Wireless Sensor Networks," Sensors, vol. 12, no. 8, pp. 11113-11153, 2012.

[24] J. Xu, N. Jin, X. Lou, T. Peng, Q. Zhou, and Y. Chen, "Improvement of LEACH protocol for WSN," 9th International Conference on Fuzzy Systems and Knowledge Discovery, pp. 2174-2177, 2012.

[25] I. Daanoune, A. Baghdad, and A. Balllouk, "BRE-LEACH: A New Approach to Extend the Lifetime Of WSN," In Third Inter. Conference on Intelligent Computing in Data Sciences (ICDS), pp. 1-6, 2019.

[26] S. B. B. Gowda and G. N. Subramanya, "DUCA: An Approach to Elongate the Lifetime of WSN," Engineering Vibration, Communication and Information Processing, pp. 329-337, 2019.

\section{BIOGRAPHIES OF AUTHORS}

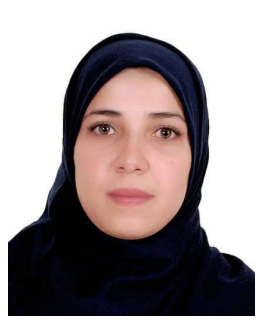

Ikram Daanoune is born on December 29, 1993, in Beni Mellal, Morocco. She obtained her license degree in electronic engineering and telecommunications, from Faculty of Sciences \& Techniques (FST) of Beni Mellal, Sultan Moulay Slimane University, in 2015, and an engineering diploma on electrical \& telecommunications at FST at Mohammedia, Hassan II University, in 2018. Presently, she is a Ph.D. student, nominee in the Electronics, Energy, Automatic and Information Processing (EEA\&TI) laboratory at FST Mohammedia, Hassan II University Casablanca. Her study interests include the development and simulation of classical and computational intelligence routing protocols to optimize energy consumption in Wireless Sensor Network.

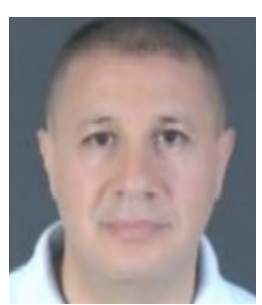

Abdennaceur Baghdad is a doctoral holder in Electronics from Lille University France in 1992. Presently, He is a university professor with PES grade at FST Mohammedia, Hassan II University Casablanca-Morocco whither he educates the hyper-frequencies, electronic engineering, antenna, and systems of telecommunication. He is a participant of the EEA\&TI laboratory at Hassan II University. His researches focused on optical communication, electronic and embedded systems, telecommunication systems and information technology. He oversees and he co-supervises many doctoral theses. $\mathrm{He}$ is a member of the organizing committees of several international congresses in the same research area.

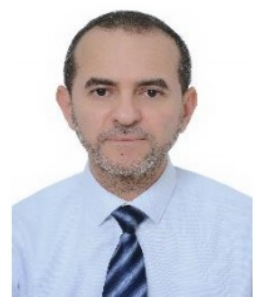

Abdelhakim Ballouk is a doctoral holder in Electronics from Bordeaux University France in 1992, in the automatic domain. Presently, He is a university professor with PES grade at the FST Mohammedia, Hassan II University Casablanca-Morocco where he educates regulation \& digital control, and linear \& non-linear automatic. He is a participant of the EEA\&TI laboratory at Hassan II University. He oversees and he co-supervises many doctoral theses. His researches concentrated on Automatic (robust commands, predictive controls, etc.). His researches focused also in Telecommunication systems (Antenna, wireless sensor network, etc.). 